\title{
Wie wirksam ist Regionalförderung?
}

\section{Céline Diebold}

\section{Relevanz}

Das Ziel der Regionalpolitik ist die Förderung strukturschwacher Gebiete, damit sie zum nationalen Durchschnitt aufschliessen. Doch die Steuermittel sind knapp und haben viele gute Verwendungen. Auch die Regionalförderung muss sich mit ihrem Nutzen rechtfertigen. Ist sie tatsächlich wirksam, die Entwicklung benachteiligter Gebiete mit zusätzlichen Investitionen anzustossen? Oder erschöpft sie sich in reinen Mitnahmeeffekten, indem sie Investitionen fördert, welche die Unternehmen ohnehin getätigt hätten? Führt sie zu einer Verlagerung von Investitionen und Beschäftigung von nicht geförderten $\mathrm{zu}$ geförderten Gebieten, ohne dass die Gesamtwirtschaft profitiert? Kann die Regionalförderung eine nachhaltige, selbsttragende Entwicklung anstossen, die auch dann noch bestehen bleibt, wenn die Förderung wieder ausläuft? Oder bleibt der neu geschaffene Wohlstand dauerhaft von Subventionen abhängig?

\section{Quelle}

Criscuolo Chiara, Ralf Martin, Henry G. Overman und John Van Reenen (2019), Some Causal Effects of an Industrial Policy, American Economic Review 109, $48-85$.

C. Diebold $(\bowtie)$

Universität St.Gallen, St.Gallen, Schweiz

E-Mail: celine.diebold@unisg.ch 
Viele Staaten setzen seit langem Subventionen und Zuschüsse ein, um wirtschaftlich benachteiligte, strukturschwache Regionen zu fördern. Die Wirksamkeit solcher Massnahmen ist allerdings umstritten. Kritiker mutmaßen, dass solche Initiativen sich in Mitnahmeeffekten erschöpfen und lediglich private Investitionen finanzieren, welche die Unternehmen ohnehin getätigt hätten.

Wie wirksam ist die Regionalförderung? Empirische Untersuchungen, inwieweit staatliche Zuschüsse Investitionen und Beschäftigung steigern können, sind rar. Ein Grund dafür liegt in der Schwierigkeit, tatsächlich den kausalen Effekt einer solchen Förderung festzumachen. Ein blosser Vergleich der wirtschaftlichen Entwicklung von Unternehmen mit und ohne staatlicher Förderung genügt jedenfalls nicht. Schließlich liegen die geförderten Unternehmen oft in strukturschwachen Regionen, was das Unternehmenswachstum im Vergleich zu anderen Regionen verzerren kann.

Chiara Criscuolo, Ralf Martin, Henry G. Overman und John Van Reenen untersuchen die Wirkungen der Regional- und Industriepolitik auf den Arbeitsmarkt in Großbritannien. Sie analysieren das Förderprogramm „Regional Selective Assistance“, welches seit den 1980er Jahren besteht und Arbeitsplätze vor allem in der Industrie und im produzierenden Gewerbe schaffen und sichern soll. Unternehmen in strukturschwachen Regionen können Zuschüsse für Investitionen von bis zu $35 \%$ der Gesamtkosten beantragen.

Um den kausalen Effekt der Regionalförderung festzumachen, nutzt das Forscherteam eine Reform aus dem Jahr 2000. Da regionale Förderprogramme den Wettbewerb verzerren können, unterliegen sie den Vorschriften der Europäischen Union. Die EU erlaubt wirtschaftliche Förderprogramme nur, wenn sie tatsächlich zur Unterstützung strukturschwacher Regionen beitragen. Solche Regionen sind in mehrere Kategorien eingeteilt, welche Investitionszuschüsse in unterschiedlicher Höhe zulassen. Diese Einteilung wird regelmäßig angepasst, so auch im Jahr 2000. Dies veränderte die Förderbarkeit der britischen Regionen sowie die zulässige Höhe der Investitionszuschüsse, welche ein lokal ansässiges Unternehmen beantragen kann.

Die Karte in Abb. 1 veranschaulicht diese Veränderungen. Vor 2000 waren gut 3400 der 10.737 Regionen Großbritanniens förderberechtigt. Der maximale Investitionszuschuss lag meist bei $20 \%$ der Gesamtkosten und bei $30 \%$ in besonders strukturschwachen Regionen. Im Zuge der Neueinstufung im Jahr 2000 verloren über 1000 Regionen die Berechtigung zu Förderungen. Dagegen wurden knapp 500 Regionen neu als strukturschwach und somit förderfähig eingestuft. Das Volumen des Förderprogrammes betrug im Beobachtungszeitraum jährlich etwa 164 Mio. Pfund. 


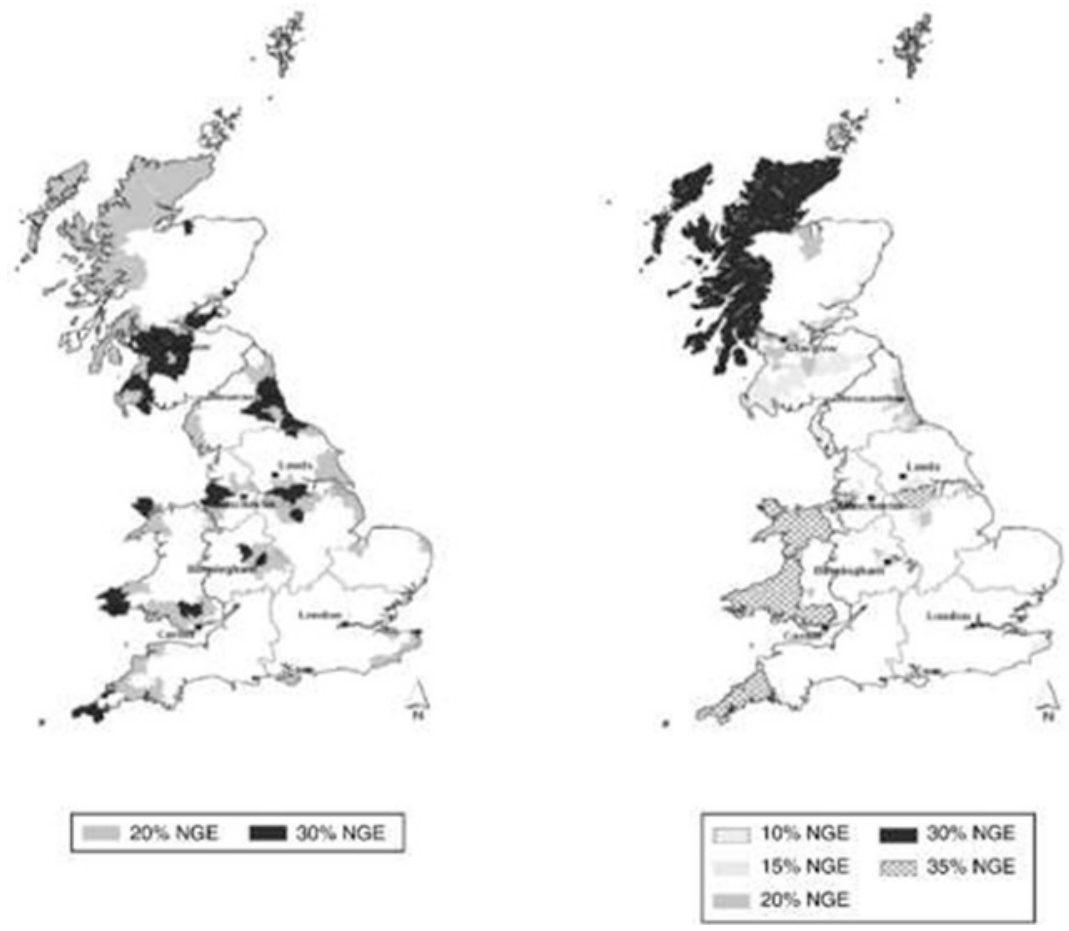

Abb. 1 Maximale regionale Investitionszuschüsse vor und nach 2000 (links vorher, rechts nachher). Bemerkung: NGE Net Grant Equivalent (zulässige Förderhöhe). (Quelle: Ciscuolo et al. 2019, S. 3)

Die Wissenschaftler untersuchen im Zeitraum von 1997 bis 2004 die Entwicklung der Beschäftigung und Arbeitslosigkeit in den verschiedenen Regionen sowie den wirtschaftlichen Erfolg der Unternehmen. Die Studie findet ökonomisch relevante Verbesserungen der Arbeitsmarktsituation. Wenn Unternehmen in einer Region einen um 10 Prozentpunkte höheren Investitionszuschuss beantragen können, erhöht dies die Zahl der Industriearbeitsplätze durchschnittlich um $10 \%$. Die Anzahl der Arbeitslosen in jener Region geht dadurch um rund 4,2\% zurück. Dieses Ergebnis gilt auch unter Berücksichtigung anderer wirtschaftspolitischer Massnahmen, welche im selben Zeitraum umgesetzt wurden. Wäre das Förderprogram im Jahr 2000 ausgelaufen, hätte dies einen hypothetischen Verlust von nahezu 156.000 Arbeitsplätzen in Großbritannien bedeutet. 
Steigt der maximale Investitionszuschuss für Industrieunternehmen um 10 Prozentpunkte, nimmt die Beschäftigung in diesem Sektor um 10 Prozent zu.

Der Beschäftigungsanstieg durch das Förderprogramm kommt in erster Linie durch die Schaffung neuer Arbeitsplätze und nicht durch die Verlagerung aus anderen Regionen oder Branchen zustande. Die Forscher finden keine Evidenz, dass die Investitionszuschüsse mit signifikanten Arbeitsplatzverlusten in anderen Regionen oder Branchen, welche nicht davon profitieren, verbunden sind.

Die positiven Beschäftigungseffekte der regionalen Förderprogramme gehen nicht mit einer Verlagerung von Arbeitsplätzen aus anderen Regionen einher.

Die Industriepolitik begünstigt in erster Linie bestehende Unternehmen. Dort entstehen die meisten der neuen Arbeitsplätze. Es sind vor allem kleine Unternehmen mit weniger als 50 Mitarbeitern, die durch Zuschüsse neue Arbeitsplätze schaffen. Große Unternehmen stellen hingegen im Durchschnitt keine zusätzlichen Mitarbeiter ein. Eine mögliche Ursache besteht darin, dass sie über mehr Spielraum verfügen, solche Programme auszunutzen. Zum Beispiel können sie leichter vorgeben, neue Arbeitsplätze zu schaffen, während sie an anderer Stelle abbauen.

Vor allem in kleineren Unternehmen führen Investitionszuschüsse zu mehr Arbeitsplätzen.

Innovation und hohe Produktivität stärken nachhaltig das Unternehmenswachstum. Die Schätzungen zeigen jedoch, dass staatliche Zuschüsse zwar die Investitionen und die Beschäftigung der Unternehmen in strukturschwachen Regionen erhöhen, jedoch ihre Produktivität nicht signifikant steigern können.

Zuschüsse führen zu einem Anstieg der Investitionen. Jedoch haben sie darüber hinaus keinen Einfluss auf die Produktivität der geförderten Unternehmen.

Die positiven Beschäftigungseffekte der Regionalförderung sind daher nicht von Dauer. Nach dem Wegfall von Förderungen, z. B. wenn eine Region die Förderbarkeit verliert, verschwinden die positiven Beschäftigungseffekte wieder. Die Einführung und Beendigung eines regionalen Förderprogramms löst in etwa gleich grosse Beschäftigungseffekte aus. Dies weist darauf hin, dass die Regionalförderung zwar zur Sicherung und Schaffung von Arbeitsplätzen beiträgt. Darüber hinaus kann sie jedoch kaum eine nachhaltig positive, selbst tragende Regionalentwicklung anstoßen. 
Open Access Dieses Kapitel wird unter der Creative Commons Namensnennung 4.0 International Lizenz (http://creativecommons.org/licenses/by/4.0/deed.de) veröffentlicht, welche die Nutzung, Vervielfältigung, Bearbeitung, Verbreitung und Wiedergabe in jeglichem Medium und Format erlaubt, sofern Sie den/die ursprünglichen Autor(en) und die Quelle ordnungsgemäß nennen, einen Link zur Creative Commons Lizenz beifügen und angeben, ob Änderungen vorgenommen wurden.

Die in diesem Kapitel enthaltenen Bilder und sonstiges Drittmaterial unterliegen ebenfalls der genannten Creative Commons Lizenz, sofern sich aus der Abbildungslegende nichts anderes ergibt. Sofern das betreffende Material nicht unter der genannten Creative Commons Lizenz steht und die betreffende Handlung nicht nach gesetzlichen Vorschriften erlaubt ist, ist für die oben aufgeführten Weiterverwendungen des Materials die Einwilligung des jeweiligen Rechteinhabers einzuholen.

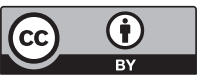

